\title{
The Irreducible Core of a Minimum Cost Spanning Tree Game
}

\author{
HARRY AARTS AND THEO DRIESSEN \\ University of Twente, P.O. Box 217, 7500 AE Enschede, The Netherlands
}

\begin{abstract}
It is a known result that for a minimum cost spanning tree (mcst) game a Core allocation can be deduced directly from a mcst in the underlying network. To determine this Core allocation one only needs to determine a mcst in the network and it is not necessary to calculate the coalition values of the corresponding mest game. In this paper we will deduce other Core allocations directly from the network, without determining the corresponding mcst game itself: we use an idea of Bird (cf. [4]) to present two procedures that determine a part of the Core (called the Irreducible Core) from the network.
\end{abstract}

Key Words: network, minimum cost spanning tree (mcst) game, Core, Irreducible Core, mcst allocation, marginal allocation.

\section{Introduction}

A mest game is a cooperative cost game that arises from a cost allocation problem in a complete weighted graph. One node of this graph represents a source that can provide the other nodes (which are identified with the players) with a certain good or service. The value of a coalition in the most game is determined by the minimal cost to provide the members of that coalition with the good or service involved, without help from the players outside the coalition, i.e., the minimal cost to connect all players in the coalition to the source (directly or via other players in that coalition). This minimal cost is precisely the cost of a mcst in the weighted subgraph induced by the coalition involved. Mcst games were introduced by Bird [4]. Throughout this paper we will use the following notations and definitions concerning mcst games.

For $n \in \mathbb{N}$ put $N:=\{1,2, \ldots, n\}$ and for $S \subseteq N$ put $S_{0}:=S \cup\{0\}$.

Definition 1.1: $A$ network on $N_{0}$ is an ordered pair $\left\langle K_{N_{0}}, w\right\rangle$, where $K_{N_{0}}:=$ $\left\langle N_{0}, E\left(K_{N_{0}}\right)\right\rangle$ represents the complete graph with node set $N_{0}$ and set of undirected edges $\left.E^{\bullet} K_{N_{0}}\right):=\left\{\{i, j\} \mid i, j \in N_{0}, i \neq j\right\}$, and $w: E\left(K_{N_{0}}\right) \rightarrow \mathbb{R}_{+}$represents a nonnegative function on $E\left(K_{N_{0}}\right)$. 
The nodes in $N$ are interpreted as the users in the network and node 0 as the common supplier. The function $w$ is called the weight function of the network. Let $2^{N}$ denote the power set of $N$, i.e., $2^{N}:=\{S \mid S \subseteq N\}$.

Definition 1.2: Let $\left\langle K_{N_{0}}, w\right\rangle$ be a network on $N_{0}$ and $S \in 2^{N} \backslash\{\varnothing\}$. The subnetwork of $\left\langle K_{N_{0}}, w\right\rangle$ on $S_{0}$ is the ordered pair $\left\langle K_{S_{0}}, w\right\rangle$, where $K_{S_{0}}:=\left\langle S_{0}, E\left(K_{S_{0}}\right)\right\rangle$ represents the complete graph with node set $S_{0}$ and edge set $E\left(K_{S_{0}}\right):=\{\{i, j\} \mid i$, $\left.j \in S_{0}, i \neq j\right\}$.

Note that the restriction of the weight function $w$ to the edge set $E\left(K_{S_{0}}\right)$ is also denoted by $w$.

Definition 1.3: The minimum cost spanning tree (mest) game corresponding to the network $\left\langle K_{N_{0}}, w\right\rangle$ is the cooperative cost game in characteristic function form $\langle N, c\rangle$, where the characteristic function $c: 2^{N} \rightarrow \mathbb{R}$ is given by

$$
c(\varnothing):=0
$$

and for all $S \in 2^{N} \backslash\{\varnothing\}$

$$
c(S):=\text { total weight of a mcst } \Gamma_{S_{0}}=\left\langle S_{0}, E\left(\Gamma_{S_{0}}\right)\right\rangle \text { in the subnetwork }\left\langle K_{S_{0}}, w\right\rangle,
$$

i.e.,

$$
c(S):=\sum\left\{w(e) \mid e \in E\left(\Gamma_{S_{0}}\right)\right\} .
$$

In the game theoretic context, elements of $N$ are called players. Mcst games were also studied in Granot and Huberman [7], [8], [9], Aarts [1] and Aarts and Driessen [2], [3]. In these papers special attention is paid to the Core of mcst games. The Core $C(c)$ of a cooperative cost game $\langle N, c\rangle$ is the set of all allocations of the joint $\operatorname{cost} c(N)$ that have the property that no coalition would be better off if it would separate and pay its cost in the game, so

$$
C(c):=\left\{x \in \mathbb{R}^{n} \mid x(N)=c(N) \text { and } x(S) \leq c(S) \text { for all } S \in 2^{N}\right\} .
$$

Here the $i$-th coordinate of $x \in \mathbb{R}^{n}$ represents the charge to player $i$ according to the allocation $x$. For any $x \in \mathbb{R}^{n}$ and $S \in 2^{N}$, we write $x(S)$ instead of $\sum\left\{x_{i} \mid i \in S\right\}$, where $x(\varnothing)=0$. Granot and Huberman [7] showed that mcst games possess a nonempty Core and their proof is based on the construction of one specific Core 
allocation. This allocation can be derived directly from a mest $\Gamma_{N_{0}}$ in the underlying network as follows: each player $i$ is charged the weight of the unique edge, incident with node $i$, that is on the path in $\Gamma_{N_{0}}$ from node $i$ to the common supplier 0 . We will denote this so-called mcst allocation corresponding to the mcst $\Gamma_{N_{0}}$ by $T\left(\Gamma_{N_{0}}\right)$.

Summarizing, for mcst games a Core allocation can be determined directly from (a mcst in) the network, i.e., it is not necessary to determine the coalition values of the corresponding mcst game (using Definition 1.3) in order to determine this particular Core allocation. A lot of efforts have been made to find Core allocations, other than mest allocations, that can also be deduced directly from the network (without first determining the mcst game itself). In case the network contains a mcst which is a chain (i.e., a spanning tree in which all nodes have degree two, except for the common supplier and one other node which have degree one), lots of additional Core allocations can be determined in this way (cf. [3], [1]). For an arbitrary network, however, the only known result is Granot and Huberman's (repeated) weak demand operation (cf. [9]). This operation transforms a mcst allocation into another allocation using the idea that, if allocated according to a mcst allocation, a player $i$ can charge his followers (in the mcst considered) some amount for using (in order to connect themselves to the common supplier) the edge that connects $i$ to his predecessor. (According to the mcst allocation the cost of this edge is fully charged to $i$ himself!) In [4] another idea is presented to obtain Core allocations from the network. In [4] a new weight function is constructed with the aid of a fixed most in the network considered. The Core of the mcst game corresponding to the new network, called the Irreducible Core, is a subset of the original Core. The main goal in this paper is to show how (the extreme points of) this Irreducible Core can be deduced from the network without first having to determine the mcst game itself.

In [4] and [6] two nice characterizations for the Irreducible Core are presented: the set of extreme points of the Irreducible Core coincides with both the set of marginal allocations of the mcst game corresponding to the adapted network and the set of all most allocations in this adapted network. We will use these characterizations to present two procedures to determine the extreme points of the Irreducible Core from this network. The first procedure determines all marginal allocations directly from this network and the second determines all mest allocations.

In the next section we will present an accurate definition of the notion of Irreducible Core and state the main known results. Then we use these results to describe the two procedures to determine this Irreducible Core from the adapted network. In the last section we elucidate one of these procedures with an example.

We conclude this section with some notations and state a graph theoretic theorem. The proof of this theorem can be found in [3].

An arbitrary graph $G$ will be denoted by the ordered pair $\langle V(G), E(G)\rangle$, where $V(G)$ represents the node (vertex) set and $E(G)$ the (undirected) edge set of $G$. If there can be no ambiguity we write $V$ and $E$ instead of $V(G)$ and $E(G)$ respec- 
tively. A (non-negative) weighted graph $G$ will be denoted by the ordered triple $\langle V(G), E(G), w\rangle$, where $w: E(G) \rightarrow \mathbb{R}_{+}$represents a non-negative weight function. Let $\Gamma=\langle V(G), E(\Gamma)\rangle$ be a spanning tree in a connected graph $G=$ $\langle V(G), E(G)\rangle$. For any $i, j \in V(G)$, we denote the unique path in $\Gamma$ from $i$ to $j$ (or vice versa) by $P_{i j}(\Gamma):=\left\langle V\left(P_{i j}(\Gamma)\right), E\left(P_{i j}(\Gamma)\right)\right\rangle$. Similarly, for any $e \in E(G)$ we denote the unique path in $\Gamma$ from one end point of $e$ to the other by $P_{e}(\Gamma):=$ $\left\langle V\left(P_{e}(\Gamma)\right), E\left(P_{e}(\Gamma)\right)\right\rangle$. In particular, $P_{e}(\Gamma)=P_{i j}(\Gamma)$ whenever $e=\{i, j\} \in E(G)$.

Theorem 1.4: The following statements for a spanning tree $\Gamma=\langle V, E(\Gamma)\rangle$ in a fixed weighted connected graph $\langle V, E, w\rangle$ are equivalent.

i) $\Gamma$ is a mcst in $\langle V, E, w\rangle$

ii) $w(e) \geq w\left(e^{\prime}\right)$ for all $e \in E \backslash E(\Gamma)$ and $e^{\prime} \in E\left(P_{e}(\Gamma)\right)$.

\section{The Irreducible Core}

In this section we define an adaptation of the weight function in a network. This adaptation was introduced in [4] to determine additional Core allocations for mcst games. We will mention some interesting properties of the resulting network and its corresponding mcst game. We will define the notion of Irreducible Core and present two procedures to determine the extreme points of this subset of the original Core directly from the adapted network.

Definition 2.1: Let $\Gamma_{N_{0}}$ be a mcst in $\left\langle K_{N_{0}}, w\right\rangle$. Define the network $\left\langle K_{N_{0}}, w^{T_{N_{0}}}\right\rangle$ by

$$
w^{\Gamma_{N_{0}}}(e):=\max \left\{w(\tilde{e}) \mid \tilde{e} \in E\left(P_{e}\left(\Gamma_{N_{0}}\right)\right)\right\} \quad \text { for all } e \in E\left(K_{N_{0}}\right)
$$

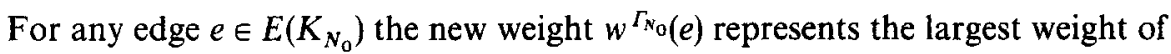
all edges that are on the path in $\Gamma_{N_{0}}$ from one endpoint of $e$ to the other. The next proposition states some elementary properties of the network $\left\langle K_{N_{0}}, w^{T_{N_{0}}}\right\rangle$.

\section{Proposition 2.2:}

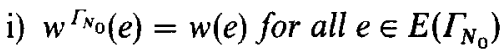

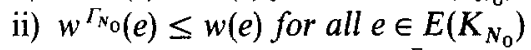

iii) $\Gamma_{N_{0}}$ is a mcst in $\left\langle K_{N_{0}}, w^{\Gamma_{N_{0}}}\right\rangle$

iv) If $\Gamma_{N_{0}}^{\prime}$ is a mcst in $\left\langle K_{N_{0}}, w\right\rangle$, then $\Gamma_{N_{0}}^{\prime}$ is also a mcst in $\left\langle K_{N_{0}}, w^{\Gamma_{N_{0}}}\right\rangle$ and

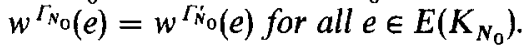


Proof:

i) follows directly from Definition 2.1 since $P_{e}\left(\Gamma_{N_{0}}\right)=\{e\}$ for all $e \in E\left(\Gamma_{N_{0}}\right)$.

ii) is a consequence of Theorem 1.4 applied to the mcst $\Gamma_{N_{0}}$ in $\left\langle K_{N_{0}}, w\right\rangle$.

iii) follows from (i), Definition 2.1 and Theorem 1.4.

iv) Let $\Gamma_{N_{0}}^{\prime}$ be a mcst in $\left\langle K_{N_{0}}, w\right\rangle$. Then

$$
\begin{aligned}
& \sum\left\{w^{\Gamma_{N_{0}}}(e) \mid e \in E\left(\Gamma_{N_{0}}^{\prime}\right)\right\} \leq \sum\left\{w(e) \mid e \in E\left(\Gamma_{N_{0}}^{\prime}\right)\right\} \\
& \quad=\sum\left\{w(e) \mid e \in E\left(\Gamma_{N_{0}}\right)\right\}=\sum\left\{w^{\Gamma_{N_{0}}}(e) \mid e \in E\left(\Gamma_{N_{0}}\right)\right\},
\end{aligned}
$$

where the inequality follows from (ii), the first equality from the fact that both $\Gamma_{N_{0}}$ and $\Gamma_{N_{0}}^{\prime}$ are mcst's in $\left\langle K_{N_{0}}, w\right\rangle$ and the last equality from (i). So $\Gamma_{N_{0}}^{\prime}$ is a spanning tree in $\left\langle K_{N_{0}}, w^{\Gamma_{N_{0}}}\right\rangle$ with less total weight than the mcst $\Gamma_{N_{0}}$ (cf. (iii)). Therefore $\Gamma_{N_{0}}^{\prime}$ is also a mcst in $\left\langle K_{N_{0}}, w^{\Gamma_{N_{0}}}\right\rangle$. This implies that the inequality above must be an equality. So, by (ii), $w^{\Gamma_{N_{0}}}(e)=w(e)$ for all $e \in E\left(\Gamma_{N_{0}}^{\prime}\right)$. This yields $w^{\Gamma_{N_{0}}^{\prime}}(e)=\left(w^{\Gamma_{N_{0}}}\right)^{\Gamma_{N_{0}}^{\prime}}(e) \leq w^{\Gamma_{N_{0}}}(e)$ for all $e \in E\left(K_{N_{0}}\right)$, where the equality follows from Definition 2.1 applied to the mcst $\Gamma_{N_{0}}^{\prime}$ in the networks $\left\langle K_{N_{0}}, w\right\rangle$ and $\left\langle K_{N_{0}}, w^{T_{N_{0}}}\right\rangle$ respectively and the fact that $w^{\Gamma_{N_{0}}}(e)=w(e)$ for all $e \in E\left(\Gamma_{N_{0}}^{\prime}\right)$, whereas the inequality follows from (ii) applied to the mcst $\Gamma_{N_{0}}^{\prime}$ in the network $\left\langle K_{N_{0}}, w^{\Gamma_{N_{0}}}\right\rangle$. Exchanging the roles of $\Gamma_{N_{0}}$ and $\Gamma_{N_{0}}^{t}$ we obtain in a similar way $w^{T_{N_{0}}(e)} \leq w^{\Gamma_{N_{0}}^{\prime}}(e)$ for all $e \in E\left(K_{N_{0}}\right)$. Therefore $w^{T_{N_{0}}}(e)=w^{\Gamma_{N_{0}}^{\prime}}(e)$ for all $e \in E\left(K_{N_{0}}\right)$.

The statements (i)-(iii) together with Theorem 1.4 imply that $w^{\Gamma_{N_{0}}}$ is the smallest weight function among weight functions of networks that contain $\Gamma_{N_{0}}$ as a mcst and whose weight functions coincide with $w$ on $E\left(\Gamma_{N_{0}}\right)$. Part (iv) states that the weight function $w^{\Gamma_{N_{0}}}$ does not depend upon the choice of the mcst $\Gamma_{N_{0}}$. This justifies the notation $\bar{w}$ instead of $w^{\Gamma_{N_{0}}}$.

Now let $\langle N, \bar{c}\rangle$ denote the mcst game corresponding to the network $\left\langle K_{N_{0}}, \bar{w}\right\rangle$. The following results and their proofs can be found in [4] and [6].

\section{Theorem 2.3:}

i) $\langle N, \bar{c}\rangle$ is a concave game, i.e.,

$$
\bar{c}(S \cup\{i\})-\bar{c}(S) \geq \bar{c}(T \cup\{i\})-\bar{c}(T) \quad \text { for all } i \in N \text { and all } S \subseteq T \subseteq N \backslash\{i\}
$$

ii) $C(\bar{c})=\operatorname{ch}\left\{T\left(\Gamma_{N_{0}}\right) \mid \Gamma_{N_{0}}\right.$ is a mcst in $\left.\left\langle K_{N_{0}}, \bar{w}\right\rangle\right\}$,

where, for any set $Q \subseteq \mathbb{R}^{n}, \operatorname{ch}(Q)$ denotes the convex hull of $Q$. Part (i), which is proved in [6], implies that the Core of $\langle N, \bar{c}\rangle$ is exactly the convex hull of all marginal allocations of $\langle N, \bar{c}\rangle$ (cf. [12]). Here the notion of a marginal allocation is defined as follows. 
Definition 2.4: Let $\langle N, c\rangle$ be an arbitrary cost game and $\theta$ a permutation of the player set $N$. The marginal allocation $m^{\theta}(c) \in \mathbb{R}^{n}$ of $\langle N, c\rangle$ corresponding to $\theta$ is given by

$$
m_{i}^{\theta}(c):=c\left(P_{i}^{\theta} \cup\{i\}\right)-c\left(P_{i}^{\theta}\right) \quad \text { for all } i \in N
$$

where $P_{i}^{\theta}:=\{j \in N \mid \theta(j)<\theta(i)\}$ represents the set of players who precede player $i$ in the $\theta$-order.

In [4], the Core of $\langle N, \bar{c}\rangle$ is called the Irreducible Core of $\langle N, c\rangle$. From Proposition 2.2 (i)-(iii) it follows that $\bar{c}(N)=c(N)$ and $\bar{c}(S) \leq c(S)$ for all $S \in 2^{N}$. Therefore the Irreducible Core of $\langle N, c\rangle$ is a subset of $C(c)$. Granot and Huberman's proof of part (ii) of Theorem 2.3 in [6] shows that for each marginal allocation of $\langle N, \bar{c}\rangle$ there exists a most $\Gamma_{N_{0}}$ in $\left\langle K_{N_{0}}, \bar{w}\right\rangle$ whose corresponding mcst allocation is exactly this marginal allocation.

On basis of the results of Theorem 2.3 we mention two ways to determine the Irreducible Core (and therefore a part of the original Core). Both ways can be used without first determining the mcst games $\langle N, c\rangle$ and $\langle N, \bar{c}\rangle$.

The first way determines all marginal allocations of $\langle N, \bar{c}\rangle$ (i.e., the extreme points of the Irreducible Core) directly from the network. This way uses the following property of $\left\langle K_{N_{0}}, \bar{w}\right\rangle$. Each mest $\Gamma_{S_{0}}$ in any subnetwork $\left\langle K_{S_{0}}, \bar{w}\right\rangle$ can be extended to a mest in the subnetwork $\left\langle K_{\left(S \cup\{i\}_{0}\right.}, \bar{w}\right\rangle$ simply by adding node $i$ to $\Gamma_{S_{0}}$ with the aid of one specific edge $(i \in N \backslash S)$. This property is a consequence of the proof of Lemma 8 in [6]. Therefore each marginal allocation $m^{\theta}(\bar{c})$ of $\langle N, \bar{c}\rangle$ can be determined by constructing a mcst $\Gamma_{N_{0}}$ in $\left\langle K_{N_{0}}, \bar{w}\right\rangle$ starting with node 0 and adding all other nodes, one by one, in the order described by $\theta$. The marginal contribution $m_{\theta(i)}^{\theta}(\bar{c})$ of a player $\theta(i)$ then corresponds to the weight of an edge with minimal weight that connects node $\theta(i)$ to the mcst on node set $\{\theta(1), \ldots, \theta(i-1)\} \cup\{0\}$. This contribution therefore equals the minimal weight of an edge connecting node $\theta(i)$ with one of the nodes in $\{\theta(1), \ldots, \theta(i-1)\} \cup\{0\}$.

The second method to determine the Irreducible Core doesn't consider marginal allocations but uses the result of Theorem 2.3(ii) directly. This method determines all mcst's in $\left\langle K_{N_{0}}, \bar{w}\right\rangle$ and deduces their mcst allocations. To apply this method one must know how to find all mcst's in $\left\langle K_{N_{0}}, \bar{w}\right\rangle$. First we formulate one of Kruskal's procedures (cf. [10]) to construct a most in a weighted connected graph.

The following procedure generates a mcst in the weighted connected graph $\langle V, E, w\rangle$.

- Choose any node $v \in V$. Put $S=\{v\}$ and $\widetilde{E}=\varnothing$.

- As long as $S \neq V$, choose an edge $\{i, j\} \in E$ with $i \in S$ and $j \in V \backslash S$ such that $w(\{i, j\})=\min \{w(\{k, l\}) \mid k \in S, l \in V \backslash S,\{k, l\} \in E\}$.

Put $S:=S \cup\{j\}$ and $\widetilde{E}:=\widetilde{E} \cup\{\{i, j\}\}$. 
This procedure constructs a mcst in $\langle V, E, w\rangle$ starting with an arbitrary node $v$, i.e., a one-node-tree, and extends this tree, step by step, by adding one node at the time, each time using one of the available edges of lowest weight. The next Theorem states that each mcst in a weighted connected graph is obtainable from procedure (2.1), where the starting point can be chosen arbitrarily.

Theorem 2.5: Let $\Gamma=\langle V, E(\Gamma)\rangle$ be a mcst in the weighted connected graph $\langle V, E, w\rangle$. Then $\Gamma$ can be constructed by procedure (2.1), starting at any node $v \in V$.

Proof: Assume the contrary. Take $v \in V$ such that $\Gamma$ cannot be constructed by starting the procedure (2.1) at $v$. We construct $\Gamma$, starting at $v$ as far as possible using procedure (2.1). This will end up with a connected graph $\Gamma_{S}=\langle S, \widetilde{E}\rangle$ with $v \in S \varsubsetneqq V$ and $\tilde{E} \varsubsetneqq E(\Gamma)$. Take $\{i, j\} \in E$ such that $i \in S, j \in V \backslash S$ and $w(\{i, j\})=$ $\min \{w(\{k, l\}) \mid k \in S, l \in V \backslash S,\{k, l\} \in E\}$. Then

$$
w(\{i, j\})<\min \{w(\{k, l\}) \mid k \in S, l \in V \backslash S,\{k, l\} \in E(\Gamma)\}
$$

because the procedure (2.1) terminated at stage $\langle S, \widetilde{E}\rangle$. Take $\left\{i^{*}, j^{*}\right\} \in E\left(P_{i j}(\Gamma)\right)$ with $i^{*} \in S$ and $j^{*} \in V \backslash S$. Then $w\left(\left\{i^{*}, j^{*}\right\}\right)>w(\{i, j\})$ because of (2.2). But then $\left\langle V,(E(\Gamma) \cup\{\{i, j\}\}) \backslash\left\{\left\{i^{*}, j^{*}\right\}\right\}\right\rangle$ would be a spanning tree in $\langle V, E, w\rangle$ with less total weight than $\Gamma$. This contradiction completes the proof of the theorem.

Theorem 2.5 guarantees that all mcst's in a network can be determined by performing the procedure (2.1) in all possible ways (starting with a fixed chosen node). Now the extreme points of the Irreducible Core can be obtained simply by deducing from each mcst in $\left\langle K_{N_{0}}, \bar{w}\right\rangle$ its mcst allocation (cf. Theorem 2.3(ii)).

The two procedures described above show that several Core allocations of a mcst game other than mcst allocations can be obtained directly from the network. In the next section we will elucidate the determination of the weight function $\bar{w}$ and the extreme points of the Irreducible Core (using the second procedure) for a special class of mest games.

\section{An Example}

In [5] relationships between mcst games and bankruptcy games are studied. In this context $0<d_{1} \leq d_{2} \leq \cdots \leq d_{n}$ denotes the sequence of claims of creditors 
$1,2, \ldots, n$ respectively in a bankruptcy situation with estate $E$. We assume that $0 \leq E<d(N)$ where, for $S \subseteq N, d(S):=\sum_{i \in S} d_{i}$ (otherwise the bankruptcy problem would not exist). Further, $D:=d(N)-E$ denotes the part of the claims that cannot be met by the estate. The bankruptcy game $\langle N, u\rangle$ corresponding to this bankruptcy situation is defined as

$$
u(S):=\max \{0, E-d(N \backslash S)\} \quad \text { for all } S \subseteq N \quad \text { (cf. [11]) }
$$

In [5] necessary and sufficient conditions on the estate and the claims are given to assure that the zero-normalization of a bankruptcy game $\langle N, u\rangle$ can be represented as the cost savings game arising from a mcst game $\langle N, c\rangle$, which means that the relationship

$$
u(S)-\sum_{i \in S} u(\{i\})=\sum_{i \in S} c(\{i\})-c(S) \quad \text { for all } S \subseteq N
$$

holds. This representation is possible if and only if either $0<D \leq d_{3}$ or there exists a $k \in\{3,4, \ldots, n-1\}$ such that $d_{k}<D \leq d_{k+1}$ and $d(K) \leq D$ (where $K:=$ $\{1,2, \ldots, k\})$. It is shown in [5] that under these conditions the mcst game $\langle N, c\rangle$ involved corresponds to the network $\left\langle K_{N_{0}}, w\right\rangle$ where, for $\{i, j\} \in E\left(K_{N_{0}}\right)$, $w(\{i, j\})$ is defined by

$$
w(\{i, j\}):= \begin{cases}2 D & \text { if } i=0, j \in N \\ D & \text { if } D \leq d_{i} \leq d_{j} \\ 2 D-d_{i} & \text { if } d_{i}<D \leq d_{j} \\ 2 D-\max \left\{0, d_{i}+d_{j}-D\right\} & \text { if } d_{i} \leq d_{j}<D\end{cases}
$$

So in particular, $c(\{i\})=w(\{0, i\})=2 D$ for all $i \in N$ and hence, relationship (3.1) reduces to

$$
\begin{aligned}
c(S)= & 2|S| D+\sum_{i \in S} u(\{i\})-u(S)=2|S| D+\sum_{i \in S} \max \{0, E-d(N \backslash\{i\})\} \\
& -\max \{0, E-d(N \backslash S)\}=2|S| D-\sum_{i \in S} \min \left\{0, D-d_{i}\right\} \\
& +\min \{0, D-d(S)\} \\
= & 2|S| D+\min \{d(S), D\}-\sum_{i \in S} \min \left\{d_{i}, D\right\} \quad \text { for all } S \subseteq N
\end{aligned}
$$

It is a known result that bankruptcy games are convex games, i.e.,

$$
u(S \cup\{i\})-u(S) \leq u(T \cup\{i\})-u(T) \text { for all } i \in N \text { and all } S \subseteq T \subseteq N \backslash\{i\}
$$


So, by (3.1), the mcst game $\langle N, c\rangle$ corresponding to the network $\left\langle K_{N_{0}}, w\right\rangle$ is concave. This class of mcst games is one of the few known classes of concave mest games. For these games (in contradistinction with arbitrary most games) we can easily determine the Core, because for concave cost games the Core coincides with the convex hull of the set of all marginal allocations (cf. [12]). This and Theorem 2.3(i) encouraged us to determine the Irreducible Core of these games in order to investigate (in future research) how the Irreducible Core of a mcst game is related to its Core in case the mcst game is already concave itself. This explains why we use this particular network to elucidate the second method described in the previous section to determine the extreme points of the Irreducible Core.

Before determining the network $\left\langle K_{N_{0}}, \bar{w}\right\rangle$ we show how to determine all mcst allocations in the network $\left\langle K_{N_{0}}, w\right\rangle$ itself. In this section we will focus on the case that there exists a $k \in\{3,4, \ldots, n-1\}$ such that $d_{k}<D \leq d_{k+1}$ and $d(K) \leq$ $D$. So $d_{i}<D$ for all $i \in K$ and $d_{i} \geq D$ for all $i \in N \backslash K$. First note that

$$
2 D-\max \left\{0, d_{i}+d_{j}-D\right\}>2 D-d_{i}>D \quad \text { for all } i, j \in K .
$$

We divide $K$ into classes of players with equal demands. Let $\tilde{d}_{1}<\tilde{d}_{2}<\cdots<\tilde{d}_{t}$ be such that $\left\{\tilde{d}_{1}, \tilde{d}_{2}, \ldots, \tilde{d}_{t}\right\}=\left\{d_{1}, d_{2}, \ldots, d_{k}\right\}$, where $t \leq k=|K|$ and let for $i=1,2, \ldots, t$ respectively, $K^{i}:=\left\{j \in K \mid d_{j}=\tilde{d}_{i}\right\}$ denote the set of all players in $K$ with claim equal to $\tilde{d}_{i}$. Note that the players in $K^{i}$ have consecutive numbers. Now by Theorem 2.5 and (3.3) we have that each mcst in $\left\langle K_{N_{0}}, w\right\rangle$ can be constructed as follows.

Start with any node $v \in N \backslash K$ (note that $N \backslash K \neq \varnothing$ because $n \in N \backslash K$ ). Then connect all other nodes in $N \backslash K$ in an arbitrary order, each by using an edge of weight $D$. This results in a spanning tree $T$ on node set $N \backslash K$. Subsequently the nodes in $K^{t}$ are connected with $T$ in an arbitrary order, i.e., each node in $K^{t}$ is connected with some node in $N \backslash K$, using an edge of weight $2 D-\tilde{d}_{t}$. Then the nodes in $K^{t-1}$ are connected with $T$ in an arbitrary order, i.e., each node in $K^{t-1}$ is connected with some node in $N \backslash K$, using an edge of weight $2 D-\tilde{d}_{t-1}$. Then the nodes in $K^{t-2}$ are connected with $T$ and so on, up to the nodes in $K^{1}$. Finally 0 is connected with an arbitrary node in $N$ by an edge of weight $2 D$.

Knowing that each mcst in $\left\langle K_{N_{0}}, w\right\rangle$ can be constructed as indicated above, we now are able to describe all mcst allocations of $\langle N, c\rangle$. If we distinguish two cases concerning the unique neighbour $j$ of 0 in the mcst $\Gamma_{N_{0}}$ considered, we can derive the following expressions.

Case 1: $j \in N \backslash K$. Then

$$
T_{i}\left(\Gamma_{N_{0}}\right)= \begin{cases}2 D-d_{i} & \text { if } i \in K \\ 2 D & \text { if } i=j \\ D & \text { otherwise }\end{cases}
$$


Case 2: $j \in K$. Let $i_{j} \in N \backslash K$ denote the unique node with $\left\{j, i_{j}\right\} \in E\left(\Gamma_{N_{0}}\right)$ (cf. construction method of $\Gamma_{N_{0}}$ described above). Then

$$
T_{i}\left(\Gamma_{N_{0}}\right)= \begin{cases}2 D-d_{i} & \text { if } i \in K \backslash\{j\} \\ 2 D & \text { if } i=j \\ 2 D-d_{j} & \text { if } i=i_{j} \\ D & \text { otherwise }\end{cases}
$$

Concerning the calculations of the marginal allocations of the mcst game $\langle N, c\rangle$ of (3.2), we remark the essential property that any coalition $S \subseteq N$ with $|S| \geq 2$ satisfies $d(S) \leq D$ if and only if $S \subseteq K$. Moreover, by (3.2), the equality

$$
c(S \cup\{i\})-c(S)=2 D+\min \{d(S \cup\{i\}), D\}-\min \left\{d_{i}, D\right\}-\min \{d(S), D\}
$$

holds for each $i \in N$ and each $S \subseteq N \backslash\{i\}$. Now it is straightforward to verify that

$$
m_{i}^{\theta}(c)= \begin{cases}2 D & \text { if } i \in P_{j_{\theta}}^{\theta} \\ 2 D-d\left(P_{i}^{\theta}\right) & \text { if } i=j_{\theta} \\ 2 D-\min \left\{d_{i}, D\right\} & \text { otherwise }\end{cases}
$$

where, for any permutation $\theta$ of $N, j_{\theta} \in N \backslash K$ satisfies $\theta\left(j_{\theta}\right) \leq \theta(l)$ for all $l \in N \backslash K$.

Notice that, if $j \in N \backslash K, T\left(\Gamma_{N_{0}}\right)$ coincides with the marginal allocation $m^{\theta}(c)$ corresponding to any permutation $\theta$ of $N$ satisfying $\theta(j)=1$. If $j \in K$ then $T\left(\Gamma_{N_{0}}\right)$ coincides with the marginal allocation $m^{\theta}(c)$ corresponding to any permutation $\theta$ of $N$ satisfying $\theta(j)=1$ and $\theta\left(i_{j}\right)=2$.

Determining the weight function $\bar{w}$ can easily be done using Definition 2.1 applied to the mcst $\left\langle N_{0},\{\{0, n\}\} \cup\{\{i-1, i\} \mid i=k+2, \ldots, n\} \cup\{\{i, k+1\} \mid\right.$ $i \in K\}>$. The result of such an exercise is

$$
\bar{w}(\{i, j\}):= \begin{cases}2 D & \text { if } i=0, j \in N \\ D & \text { if } D \leq d_{i} \leq d_{j} \\ 2 D-d_{i} & \text { if } d_{i}<D \leq d_{j} \text { or } d_{i} \leq d_{j}<D\end{cases}
$$

By Theorem 2.5 and $2 D>2 D-d_{i}>D$ for all $i \in K$ we have that each mcst in $\left\langle K_{N_{0}}, \bar{w}\right\rangle$ can be constructed as follows.

Start with any node $v \in N \backslash K$. Then connect all other nodes in $N \backslash K$ in an arbitrary order, each by using an edge of weight $D$. Subsequently, connect all nodes in $K^{t}$, one by one, in an arbitrary order, using an edge of weight $2 D-\tilde{d}_{t}$ (so the first node of $K^{t}$ (in the chosen order) is connected with some node in $N \backslash K$, the others with either a node in $N \backslash K$ or a node in $K^{t}$ which is already connected). 
Then connect all nodes in $K^{t-1}$, one by one, in an arbitrary order, using an edge of weight $2 D-\tilde{d}_{t-1}$ (so the first node of $K^{t-1}$ (in the chosen order) is connected with some node in $(N \backslash K) \cup K^{t}$, the others with either a node in $(N \backslash K) \cup K^{t}$ or a node in $K^{t-1}$ which is already connected). Then the nodes in $K^{t-2}$ are connected and so on, up to the nodes in $K^{1}$. Finally 0 is connected to an arbitrary node in $N$ by an edge of weight $2 D$.

Knowing the structure of the mcst's in $\left\langle K_{N_{0}}, \bar{w}\right\rangle$, we now can describe all mcst allocations of $\langle N, \bar{c}\rangle$. Let again $j \in N$ denote the unique neighbour of 0 in the most $\Gamma_{N_{0}}$ considered.

Case 1: $j \in N \backslash K$. Now the structure of $\Gamma_{N_{0}}$ directly implies that

$$
T_{i}\left(\Gamma_{N_{0}}\right)= \begin{cases}2 D-d_{i} & \text { if } i \in K \\ 2 D & \text { if } i=j \\ D & \text { otherwise }\end{cases}
$$

Case 2: $j \in K$, say $j \in K^{i}\left(i \in\{1, \ldots, t\}\right.$ ). Consider the (unique) path in $\Gamma_{N_{0}}$ from $j$ to some node $u$ in $N \backslash K$. Put $l_{1}:=j$ and let $\left\{j_{1}, l_{2}\right\},\left\{j_{2}, l_{3}\right\}, \ldots,\left\{j_{p}, l_{p+1}\right\}$ denote the edges in $E\left(P_{j u}\left(\Gamma_{N_{0}}\right)\right)$ that satisfy $j_{r} \in K^{i_{r}}$ and $l_{r} \in K^{i_{r}}$ for all $1 \leq r \leq p$ and $l_{p+1} \in N \backslash K$, where $i=i_{1}<i_{2}<\cdots<i_{p} \leq t$ (the edges $\left\{j_{1}, l_{2}\right\},\left\{j_{2}, l_{3}\right\}, \ldots$, $\left\{j_{p}, l_{p+1}\right\}$ denote those edges on the path $P_{j u}\left(\Gamma_{N_{0}}\right)$ that connect nodes corresponding to players with different claims. Put, for $1 \leq r \leq p, L^{i_{r}}:=K^{i_{r}} \cap$ $V\left(P_{i j_{p}}\left(\Gamma_{N_{0}}\right)\right.$ ) (cf. Figure 3.1)). Now we can deduce immediately that $T_{j}\left(\Gamma_{N_{0}}\right)=2 D$ and, for $1 \leq r \leq p, T_{l_{r+1}}\left(\Gamma_{N_{0}}\right)=2 D-\tilde{d}_{i_{r}}=2 D-d_{j_{r}}$. If $i \in N \backslash\left(K \cup\left\{l_{p+1}\right\}\right)$ then $E\left(P_{i l_{p+1}}\left(\Gamma_{N_{0}}\right)\right) \cap E\left(P_{l_{p+1} 0}\left(\Gamma_{N_{0}}\right)\right)=\varnothing$ because $V\left(P_{i l_{p+1}}\left(\Gamma_{N_{0}}\right)\right) \subseteq N \backslash K$ (cf. construction of $\Gamma_{N_{0}}$ described above) and therefore we deduce from $E\left(P_{i 0}\left(\Gamma_{N_{0}}\right)\right)=$ $E\left(P_{i l_{p+1}}\left(\Gamma_{N_{0}}\right)\right) \cup E\left(P_{l_{p+1} 0}\left(\Gamma_{N_{0}}\right)\right)$ that $T_{i}\left(\Gamma_{N_{0}}\right)=D$. Now it remains to consider the case $i \in K \backslash\left\{l_{1}, \ldots, l_{p}\right\}$. If $i \in V\left(P_{j j_{p}}\left(\Gamma_{N_{0}}\right)\right) \backslash\left\{l_{1}, \ldots, l_{p}\right\}$, say $i \in L^{i_{r}}$ for some $1 \leq r \leq$ $p$, then $T_{i}\left(\Gamma_{N_{0}}\right)=2 D-\tilde{d}_{i_{r}}=2 D-d_{i}$. If $i \in K \backslash V\left(P_{j j_{p}}\left(\Gamma_{N_{0}}\right)\right)$ then the inclusion $E\left(P_{i 0}\left(\Gamma_{N_{0}}\right)\right) \subseteq E\left(P_{i v}\left(\Gamma_{N_{0}}\right)\right) \cup E\left(P_{v u}\left(\Gamma_{N_{0}}\right)\right) \cup E\left(P_{u 0}\left(\Gamma_{N_{0}}\right)\right)$ (the edge set of the path

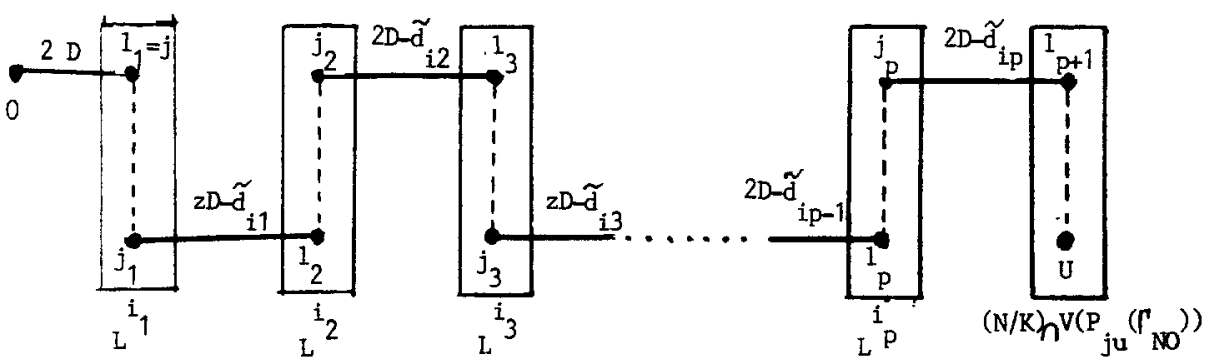

Fig. 3.1. Sketch of $P_{O u}\left(\Gamma_{N_{0}}\right)$ 
from $i$ to 0 is contained in the edge set of any walk from $i$ to 0 ) together with $V\left(P_{v u}\left(\Gamma_{N_{0}}\right)\right) \subseteq N \backslash K$ (cf. construction of $\Gamma_{N_{0}}$ described above) imply that the edge in $E\left(P_{i 0}\left(\Gamma_{N_{0}}\right)\right)$ incident with $i$ coincides with the edge in $E\left(P_{i v}\left(\Gamma_{N_{0}}\right)\right)$ incident with $i$. Therefore, by the construction of $\Gamma_{N_{0}}, T_{i}\left(\Gamma_{N_{0}}\right)=2 D-d_{i}$. Summarizing,

$$
T_{i}\left(\Gamma_{N_{0}}\right)= \begin{cases}2 D & \text { if } i=j \\ 2 D-d_{j_{r}} & \text { if } i=l_{r+1}, 1 \leq r \leq p \\ D & \text { if } i \in N \backslash\left(K \cup\left\{l_{p+1}\right\}\right) \\ 2 D-d_{i} & \text { otherwise }\end{cases}
$$

Now the Irreducible Core can be obtained immediately from Theorem 2.3(ii). Notice that the set of mcst allocations of $\langle N, c\rangle$ is indeed a subset of the set of mest allocations of $\langle N, \bar{c}\rangle$. In case $t \geq 2$ this inclusion is strict, which means that in this case several Core allocations of $\langle N, c\rangle$, other than mest allocations of $\langle N, c\rangle$, are determined.

\section{References}

[1] Aarts $\mathrm{H}$ (1992) Marginal allocations in the core of minimal chain games. Working paper no. 1103, Dept. of Applied Mathematics, University of Twente, Enschede, The Netherlands

[2] Aarts H, Driessen Th (1990) A survey on minimum cost spanning tree games. Working paper no. 1003, Dept. of Applied Mathematics, University of Twente, Enschede, The Netherlands

[3] Aarts H, Driessen Th (1992) On the core-structure of minimum cost spanning tree games. Working paper no. 1085, Dept. of Applied Mathematics, University of Twente, Enschede, The Netherlands

[4] Bird CG (1976) On cost allocation for a spanning tree: A game theoretic approach. Networks $6: 335-350$

[5] Driessen Th (1992) Relationships between bankruptcy games and minimum cost spanning tree games. Working paper no. 1058, Dept. of Applied Mathematics, University of Twente, Enschede, The Netherlands

[6] Granot D, Huberman G(1977) The relationship between convex games and minimal spanning tree games: A case for permutationally convex games. Working paper, Faculty of Commerce and Business Administration, University of British Columbia, Vancouver, BC, Canada (October 1977; Revised: June 1978; June 1979)

[7] Granot D, Huberman G (1981) Minimum cost spanning tree games. Mathematical Programming 21:1-18

[8] Granot D, Huberman G (1982) The relationship between convex games and minimum cost spanning tree games: A case for permutationally convex games. SIAM Journal of Algebraic and Discrete Methods 3:288-292

[9] Granot D, Huberman G (1984) On the core and nucleolus of minimum cost spanning tree games. Mathematical Programming 29:323-347

[10] Kruskal JB Jr. (1956) On the shortest spanning subtree of a graph and the Traveling Salesman Problem. Proc Amer Math Soc 7:48-50

[11] O'Neill B (1982) A problem of rights arbitration from the Talmud. Mathematical Social Sciences 2:345-371

[12] Shapley LS (1971) Cores of convex games. International Journal of Game Theory 1:11-26 